\section{Strategies against Xalkori resistance}

\author{
By Kai-Jye Lou, Staff Writer
}

Two U.S. groups have uncovered multiple mechanisms in ALK-rearranged lung cancers that could drive resistance to Pfizer Inc.'s Xalkori crizotinib. ${ }^{1,2}$ The findings suggest Xalkori should be combined with drugs that target other oncogenic pathways such as the EGFR and KIT pathways, but future studies are needed to determine the frequencies of the identified resistance mechanisms and the best ways to overcome them.

Xalkori is a dual inhibitor of c-Met receptor tyrosine kinase (c-Met) and anaplastic lymphoma kinase (ALK), and their oncogenic variants. Last August, the FDA granted Xalkori accelerated approval to treat advanced or metastatic ALK-positive non-small cell lung cancer (NSCLC).

Pfizer originally pursued Xalkori for its c-Met inhibition properties but ended up positioning the drug for ALK-positive lung cancers after two key developments suggested an $A L K$ fusion gene was actually the oncogenic driver in a subset of patients with NSCLC.,34

The fusion gene, which contains parts of echinoderm microtubule associated protein like 4 (EML4) and ALK, encodes a cytoplasmic protein with constitutive kinase activity.

In patients with ALK-positive NSCLC, Xalkori has produced overall response rates of more than $50 \%$. In contrast, the overall response rate for second-line chemotherapies in lung cancer is only about $10 \%$.

"Crizotinib is one of the most exciting advances in lung cancer in recent years," said Jeffrey Engelman, director of the Center for Thoracic Cancers at Massachusetts General Hospital Cancer Center and an assistant professor of medicine at Harvard Medical School. "However, despite the high response rate, we find that patients who initially respond to crizotinib usually become resistant to the therapy, and half will become resistant within one year into treatment. We hope that understanding the mechanisms that drive resistance will help give us new insights on how to better treat these patients to overcome resistance or prevent it from developing in the first place."

To elucidate the mechanisms underlying Xalkori resistance, Engelman and colleagues assayed tumor biopsy samples taken from patients with $A L K$-rearranged NSCLC before and after resistance developed.

Analysis of samples from 18 patients with resistance to Xalkori showed that tumors from 5 patients had genomic alterations in $A L K$ itself -4 distinct mutations in the tyrosine kinase domain and 1 amplification of the $A L K$ fusion gene. However, other patients had epidermal growth factor receptor (EGFR) activation, and two samples showed amplification of stem cell factor receptor tyrosine kinase (c-Kit; KIT; CD117).
"We found that only about $25 \%$ of the patients with resistance developed mutations or amplifications in $A L K$ itself," said Engelman. "We did not identify $A L K$ mutations in the other $75 \%$ of patients who have developed resistance to crizotinib, which suggests that these cancers do not require $A L K$ mutations to become resistant to the drug."

In $A L K$-rearranged human lung cancer cell lines with EGFR- or KIT-mediated Xalkori resistance, Xalkori plus an EGFR or KIT inhibitor decreased proliferation compared with Xalkori alone.

Taken together, the findings suggest the mechanisms that contribute to Xalkori resistance in ALK-rearranged NSCLC are diverse and that most such mechanisms do not involve alterations to $A L K$ itself.

Results were published in Science Translational Medicine.

\section{Layers of complexity}

In a separate study, Robert Doebele and colleagues at the University of Colorado Denver School of Medicine assayed tumor biopsy samples from $14 A L K$-rearranged NSCLC patients who showed disease progression while receiving Xalkori.

Like Engelman's team, the Colorado group identified genetic changes in $A L K$ and EGFR in patients who developed resistance to Xalkori. In addition, the group detected tumors that lacked an $A L K$ gene fusion in three of the patients, which suggests the emergence of tumors driven by non- $A L K$ oncogenic pathways. The group also found two cases with activating $K$-Ras mutations.

It is not yet clear whether the $A L K$-negative and $K$-Ras-mutant tumors emerged directly or independently from the $A L K$-rearranged tumors.

"One possibility is that the cancer cells are losing ALK and gaining another oncogenic pathway," said Doebele, who is an assistant professor in the Division of Medical Oncology at the university's School of Medicine. "Another possibility is that the patient may already have multiple populations of cancer cells with independent oncogenic drivers."

Data were published in Clinical Cancer Research.

"The findings from these two
"The findings from these two studies represent an important step to understanding ALK in lung cancer biology and show that there is an extra layer of complexity in Xalkori resistance on top of mutations in ALK itself." -Timothy Clackson, Ariad Pharmaceuticals Inc. studies represent an important step to understanding ALK in lung cancer biology and show that there is an extra layer of complexity in Xalkori resistance on top of mutations in $A L K$ itself," said Timothy Clackson, president of R\&D and CSO at cancer company Ariad Pharmaceuticals Inc.

He added, "The studies suggest that resistance mechanisms in $A L K$ driven lung cancers, at least for crizotinib, are more complicated than what we typically see in $B C R-A B L$-driven CML [chronic myelogenous leukemia] and $E G F R$-driven cancers," where resistance typically results from mutations in the receptor tyrosine kinases that are being targeted by the drugs. 


\section{Getting testy}

The diversity of Xalkori resistance mechanisms suggests repeated tumor profiling in patients with $A L K$-rearranged NSCLC will be important during treatment with the drug.

Xalkori was approved concurrently with the Vysis ALK Break Apart FISH Probe test from Pfizer's partner Abbott Laboratories. The companion molecular diagnostic detects rearrangements involving the ALK gene.

But tackling the resistance problem would likely require a separate diagnostic that can screen patient tumors for a series of mechanisms that can drive resistance to Xalkori, including those that do not stem from $A L K$ itself.

"It's one thing to look at a single gene for resistance mechanisms, but these studies suggest the need to probe entire pathways with molecular diagnostics, which is going to be more complicated and raises the bar for the design of such diagnostics," said Clackson.

For Xalkori resistance mediated by mutations in the ALK kinase domain, Engelman's team reported that distinct $A L K$ mutations conferred differential sensitivities to second-generation ALK inhibitors.

"Sequencing of the $A L K$ gene in tumor DNA will be an important diagnostic method to guide therapeutic decisions and to diagnose resistance mechanisms in the future," said Tesaro Inc. president and CSO Mary Lynne Hedley. "These findings suggest the need to isolate and reanalyze patient samples as tumors begin to progress on Xalkori and provide impetus for generating improvements in blood-based and gene-sequencing diagnostics to select the best sequence for treatment with Xalkori and other ALK inhibitors that gain regulatory approval."

In March 2011, Tesaro in-licensed Amgen Inc.'s preclinical ALK inhibitor program.

\section{Strategic insights}

In addition to diagnostic insights, the resistance mechanisms also could aid the development of new ALK inhibitors.

"Now that we have identified some of these mutations, identifying ALK inhibitors that are not subject to such mutations would likely be helpful," said James Christensen, senior director of precision medicine in the division of oncology research at Pfizer. "From a structure-based design perspective, it could be helpful to design inhibitors that block the mutant forms of ALK."

"It will also be important to determine whether going in as early as possible with a more potent and more mutation-resistant inhibitor would yield improved patient outcomes in terms of survival and delayed development of resistance," Clackson told SciBX.

Because there are many $A L K$ mutations that could drive resistance, Doebele thinks companies will need to design inhibitors of multiple mutant forms of the target.

"And for tumors that have become partially or completely independent of ALK, one will need to think of combination approaches with other inhibitors such as those targeting the EGFR, KIT or K-Ras pathways," he added.

Pfizer has an ongoing Phase I/II study of Xalkori plus the EGFR inhibitor Tarceva erlotinib in patients with NSCLC. Although the new papers suggest blocking EGFR could overcome Xalkori resistance, the pharma's original rationale for the clinical trial was that Xalkori would help overcome resistance to EGFR inhibitors.

"Our rationale for combining the two drugs was that Xalkori might be able to help overcome or preemptively circumvent the development of
c-Met-mediated resistance to EGFR inhibitors," said Christensen. "One thing we're also interested in, initially from a nonclinical perspective, is alternative dosing schedules for Xalkori itself. For example, can you effectively deliver the drug by pulsing it on an intermittent schedule, and will this help delay the development of resistance?"

The findings also could open up new therapeutic avenues for Ariad's AP26113, a dual inhibitor of ALK and EGFR that is in a Phase I/II trial to treat lung cancer. The company plans to start the Phase II portion of the trial this year.

"When we first discovered AP26113, we did not anticipate that its ability to inhibit EGFR could potentially help overcome the mechanisms that drive resistance to ALK inhibition," Clackson said. "We originally selected AP26113 for development because it had potential in both EGFR- and ALKdriven lung cancers, which are generally viewed as two separate disease subtypes."

\section{Sizable samples}

Engelman said his group has expanded its ongoing study to include samples from another 10-12 patients. His group also is working with collaborators to investigate the potential of combining ALK inhibitors with other therapeutics to overcome EGFR- and KIT-mediated resistance to Xalkori.

Finally, the group is profiling how the development of resistance to one ALK inhibitor correlates with the efficacy of subsequent therapeutic agents, including other ALK inhibitors.

"It will be interesting to determine whether the use of more potent ALK inhibitors will lead to the development of a different spectrum of point mutations in ALK itself," Engelman told SciBX.

Doebele said the Colorado group is now trying to overcome the identified resistance mechanisms or prevent them from developing in the first place. He said the group also is searching for and cataloging new resistance mutations and looking at tissue samples for evidence of multiple resistance mechanisms in individual patients.

The findings reported in both papers are unpatented.

Lou, K.-J. SciBX 5(7); doi:10.1038/scibx.2012.167

Published online Feb. 16, 2012

\section{REFERENCES}

1. Katayama, R. et al. Sci. Transl. Med.; published online Jan. 25, 2012; doi:10.1126/scitransImed.3003316

Contact: Jeffrey A. Engelman, Massachusetts General Hospital Cancer Center, Boston, Mass. e-mail: jengelman@partners.org

2. Doebele, R.C. et al. Clin. Cancer Res.; published online Jan. 10, 2012; doi:10.1158/1078-0432.CCR-11-2906

Contact: Robert C. Doebele, University of Colorado Denver School of Medicine, Aurora, Colo.

e-mail: robert.doebele@ucdenver.edu

3. McCallister, E. BioCentury 19(18), A8-A9; April 25, 2011

4. Soda, M. et al. Nature 448, 561-566 (2007)

\section{COMPANIES AND INSTITUTIONS MENTIONED}

Abbott Laboratories (NYSE:ABT), Abbott Park, III. Amgen Inc. (NASDAQ:AMGN), Thousand Oaks, Calif. Ariad Pharmaceuticals Inc. (NASDAQ:ARIA), Cambridge, Mass. Harvard Medical School, Boston, Mass. Massachusetts General Hospital Cancer Center, Boston, Mass. Pfizer Inc. (NYSE:PFE), New York, N.Y.

Tesaro Inc., Boston, Mass.

University of Colorado Denver School of Medicine, Aurora, Colo. 